\title{
The study of incommensurate structures as a probe to reveal atomic interactions in crystals
}

\author{
Gervais Chapuis* and Alla Arakcheeva \\ Ecole Polytechnique Fédérale de Lausanne, Laboratoire de cristallographie, BSP, CH-1015 Lausanne, Switzerland
}

Received March 25, 2004; accepted June 4, 2004

Incommensurate structure / Molecular dynamical simulation / Superspace formalism / Sodium carbonate /

Potassium indium phosphate

\begin{abstract}
The structure of aperiodic crystals which includes incommensurate, quasi- and composite crystals is usually described in spaces of higher dimension, the so called superspace. The main advantage of the superspace formalism is that an aperiodic structure in three dimensions recovers its full periodicity in higher dimensions. The symmetry properties of aperiodic crystals are obviously more convenient to describe in superspace too. The origin of the incommensurate nature of structures can often be found in competing interatomic interactions. From molecular dynamics simulation of a simple three dimensional model with close-packed layers and a single degree of freedom for each particle, it is possible to find the existence conditions of commensurate and incommensurate phases. Incommensurate phases can already be predicted on the basis of nearest and next nearest neighbour particle interactions only. We illustrate this principle of interactions with two examples of structures, $\mathrm{Na}_{2} \mathrm{CO}_{3}$ and $\mathrm{K}_{3} \mathrm{In}\left(\mathrm{PO}_{4}\right)_{2}$. These examples shows clearly the importance of non-oxygen interactions i.e. next nearest interactions for the formation of incommensurate structures.
\end{abstract}

\section{Introduction}

The study of aperiodic crystals was initiated many decades ago when crystallographers realised that some specific diffraction patterns could not be conventionally indexed with three integers, i.e. the three coefficients associated to the three dimensional scattering vector. The appearance of additional reflections, so called satellite reflections, was already noted in the forties in some alloys (Daniel and Lipson, 1944; Hargreaves, 1951) and later in the silicate mineral labradorite. Korekawa (1967) presented a theory of satellite reflections relating their presence to some specific deformation and in particular modulations of the crystalline structure. In his work, he also presented the basic principles to deduce the structural characteristics

\footnotetext{
* Correspondence author (e-mail: gervais.chapuis@epfl.ch)
}

from the diffraction pattern. All these studies were essentially concerned with the origin of the satellite reflection but did not address the field of symmetry which is so fundamental in any crystallographic study. An important breakthrough was established when de Wolff $(1974,1977)$ introduced the idea to describe modulated structures and their symmetry in spaces of higher dimensions. The main advantage of this method was to recover the periodicity of the structure which is lost in the three-dimensional space. In the meantime, the field of modulated crystals and its generalisation, aperiodic crystals, developed up to the point that aperiodic structures have been identified and described in all type of materials, from minerals to metals and from organic to inorganic crystal structures.

In this article, we shall focus on the contribution to crystal chemistry resulting from the study of aperiodic crystals. We shall however limit the scope of our study to incommensurately modulated crystal structures. In particular, we shall show how the embedding and the description of modulated crystal structures in higher dimensional space, i.e. the superspace can help us to explain the origin of the departure from periodicity and thus contribute to a better understanding of interatomic interactions in general. In a first section, we shall explain shortly the method of crystal embedding in superspace and how the periodicity of aperiodic crystals in three dimensions can be recovered in higher dimensional space. In a second section, we shall show by molecular dynamical simulations what kind of conditions the potential function must satisfy in order to allow the existence of aperiodic structures. In the next two sections, we shall present the incommensurate structures of two parent compounds from the structural point of view, $\mathrm{Na}_{2} \mathrm{CO}_{3}$ and $\mathrm{K}_{3} \mathrm{In}\left(\mathrm{PO}_{4}\right)_{2}$ and show the importance of non-oxygen atom interactions which can be inferred from the analysis of the structures in superspace.

\section{The description of incommensurately modulated structures in the superspace formalism}

The main characteristic of aperiodic crystals and consequently incommensurate crystals is that their diffraction pattern consists essentially of discrete intensities but which 
cannot be indexed with three integers only. The idea of de Wollf (1974) was to extend the number of rationally independent vectors beyond three increasing thus the number of integer indices to describe the full spectrum of reciprocal vectors associated with the diffracted intensities. Each reciprocal vector can thus be expressed by

$$
\mathbf{H}=h_{1} \mathbf{a}_{1}^{*}+h_{2} \mathbf{a}_{2}^{*}+\ldots+h_{i} \mathbf{a}_{i}^{*}
$$

where $i$ is the minimum number of indices which are needed to index all the diffraction peaks. This number can vary in general between four and six and is called the rank of the superspace. If in the present context, we shall limit the rank to four and define the following expression

$$
\mathbf{a}_{4}^{*} \equiv \mathbf{q}=q_{1} \mathbf{a}_{1}^{*}+q_{2} \mathbf{a}_{2}^{*}+q_{3} \mathbf{a}_{3}^{*}
$$

where at least one of the $q_{i}$ is irrational. Following de Wolff, we illustrate in direct space the description of aperiodic crystals in superspace of dimension equal to the rank. Fig. 1 illustrates the embedding of the crystal structure in higher dimensional space.

We can observe that the higher dimensional structure with cell parameters $\mathbf{a}_{\mathrm{Si}}$ repeat the motif (here a continuous string with arbitrary shape) periodically. The intersecting horizontal line $R$ represents a three dimensional cut of the hypercrystal. In other words, the position of each atom is given by the intersection of the string with the horizontal line $R$. On the figure, we can observe the position of the atoms $A, B$ and $C$ in adjacent cells with dimension $a_{1}$. We observe in particular that the (interatomic) distance $A B$ is different from $B C$. This intersection is not periodic if $q_{1}$ is irrational, whereas it is periodic if $q_{1}$ is rational. On the same figure, we can also see that the periodic string (the

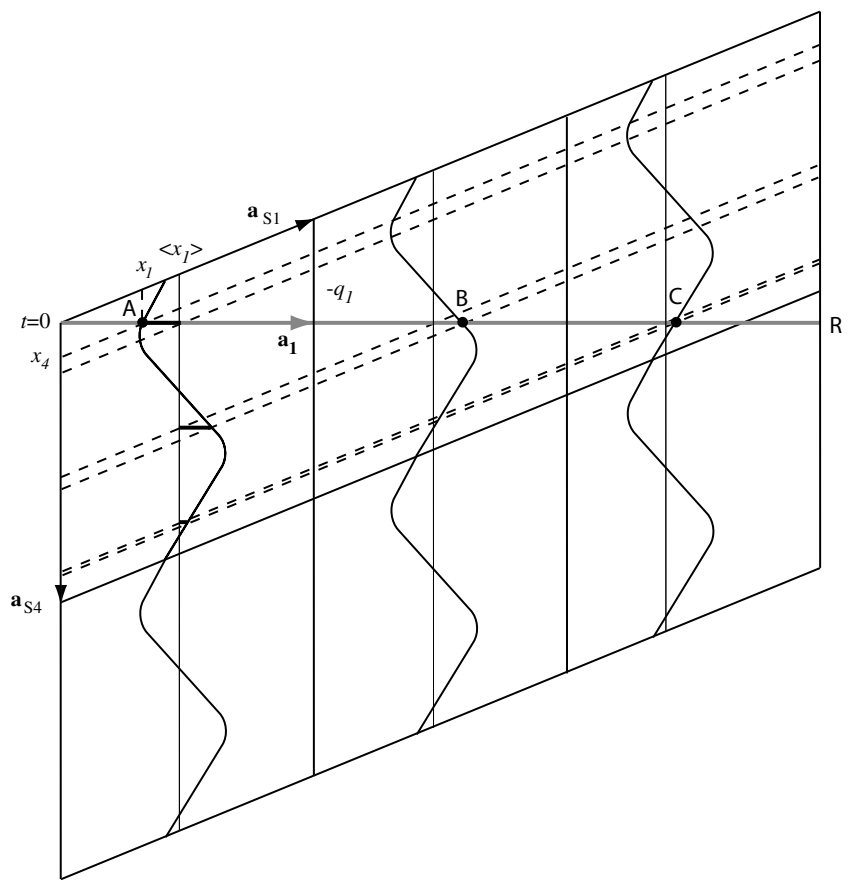

Fig. 1. The embedding of an aperiodic crystal in superspace. $\mathbf{a}_{\mathrm{S} 1}$ and $\mathbf{a}_{\mathrm{S} 4}$ are cell parameters in superspace, $x_{1}$ and $x_{4}$ are components of the coordinates of a point in superspace, $q_{1}$ is the first component of the modulation vector $\mathbf{q}$. The horizontal line $R$ (here at at $t=0$ ) represents a three dimensional cut of the superspace, i.e. the real crystal. modulation function) represents the mapping of all the displacements in each individual cell from the average position $\left\langle x_{1}\right\rangle$. In other words, if we can deduce from the experimental measurements the shape of the string for each individual atom, we can completely characterize the nature of the aperiodic structure for all atoms. If the line $R$ characterized by the parameter $t=0$ is shifted in the direction $\mathbf{a}_{\mathrm{S} 4}$ with values of $t \neq 0$, the corresponding aperiodic structure will be the same but with a shifted origin. Note that the modulation function is periodic with period $t=1$. We see here all the advantages that we can gain by exploiting the superspace formalism. For the analysis of the structure, we need only to concentrate on the hyper unit cell in superspace delimited by the vectors $\mathbf{a}_{\mathrm{Si}}$.

Since a few decades, numerous examples of incommensurate structures have been analyzed and published in the specialized literature. Each atom is characterized by a modulation function depending on $x_{4}$. However, for practical purpose it is preferable to express the modulation in term of $t$ owing to the property that the physical structure is given by an intersection with constant $t$ (see Fig. 1).

The interested reader may consult a complete overview on the subject published by Yamamoto (1996). The methods of structure analysis of incommensurate and composite crystals are also presented in this issue by Petřícek and Dušek (2004). The large number of published examples of incommensurate structures is thus a very rich source of information in order not only to understand the nature and origin of the incommensurate structures but also to get a deeper understanding of the interatomic interactions occuring in crystalline structures and during phase transitions.

Before going into the details of some examples of incommensurate structures, we shall attempt to estimate the conditions under which an incommensurate structure can exist. For this purpose, we shall use the methods of molecular dynamics and find the conditions that the potential function must satisfy. With this knowledge, we shall be in a better position to analyze the experimental data and deduce the fundamental interactions leading to the incommensuration of crystal structures.

\section{Molecular dynamical simulations of incommensurate structures}

In molecular dynamics (MD), the movement of every particle in a large physical system is subject to the classical equation of motion, the Newton equation:

$$
m_{i} \ddot{\mathbf{x}}_{i}=\mathbf{F}_{i}=-\frac{\partial V\left(\mathbf{x}_{i}\right)}{\partial \mathbf{x}_{i}}
$$

$\mathbf{x}_{i}, m_{i}$ and $\mathbf{F}_{i}$ are the position, mass and force of particle $i$. $V\left(\mathbf{x}_{i}\right)$ is the potential energy of the particle. The trajectories of the particles are obtained by numerical integration of Newton's equation. In general, this integration is usually obtained from a simple central difference procedure which gives reasonable accuracy. Time derivatives are thus replaced by finite differences with arbitrary interval $\triangle t$. The optimal choice of $\triangle t$ is a matter of considerable attention and must be evaluated for each system. In 
general, $\triangle t$ must be small as compared to the shortest normal period of the system. Starting with a complete set of positions $\mathbf{x}_{i}(t)$ at arbitrary time $t$ and the corresponding velocities $\dot{\mathbf{x}}_{i}\left(t-\frac{\triangle t}{2}\right)$, the method evaluate the new positions and velocities at $t+\triangle t$ resp. $t+\frac{\triangle t}{2}$. The process is then iterated a large number of times until it reaches an equilibrium. The macroscopic properties of the system are obtained from the average over the configurations thus obtained. As an example, the temperature of the system with $\mathrm{N}$ particles is related to the kinetic energy with the equipartition theorem:

$$
\left\langle\sum_{i} \frac{m_{i} \dot{\mathbf{x}}_{i}^{2}}{2}\right\rangle=\frac{3}{2} N k T .
$$

\section{Application to incommensurate systems}

We present here the results of a MD simulation based on a hexagonal model of particles with one particle per unit cell and one degree of freedom for each particle. The model has the shape of a rhombic prism consisting of $60 \times 60 \times 30=108000$ particles in the $a, b$ respectively $c$ directions. Each particle is only allowed to move in the $z$ direction and interacts with its first and second nearest neighbours as indicated on Fig. 2. The potential energy has the following form

$$
\begin{aligned}
V= & \frac{1}{2} \sum_{j, l, n} A z_{j, l, n}^{2}+B z_{j, l, n}\left(z_{j+1, l, n}+z_{j-1, l, n}+z_{j, l+1, n}\right. \\
& \left.+z_{j, l-1, n}+z_{j+1, l+1, n}+z_{j-1, l-1, n}\right) \\
& +z_{j, l, n}\left(z_{j+1, l-1, n}+z_{j-1, l+1, n}+z_{j+2, l+1, n}\right. \\
& \left.+z_{j-2, l-1, n}+z_{j+1, l+2, n}+z_{j-1, l-2, n}\right) \\
& +C z_{j, l, n}\left(z_{j, l, n+1}+z_{j, l, n-1}\right)+H z_{j, l, n}^{3}+z_{j, l, n}^{4} .
\end{aligned}
$$

This potential function is characterised by the three harmonic parameters $A, B$ and $C$ and the anharmonic parameter $H$. We note that the anharmonic terms are local only and that two coefficients have been set equal to one by appropriate choice of the units (Parlinski et al. 1992; Parlinski and

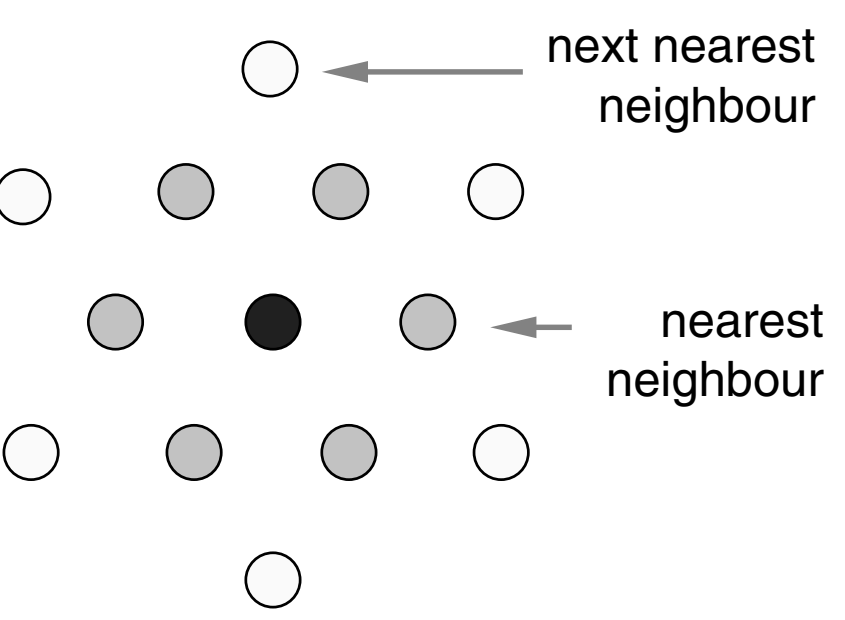

Fig. 2. Each particle is interacting with six nearest and six next nearest neigbours. In addition, each particle is also interacting with the two neighbours above and below the plane.
Chapuis, 1993). The simulations have been performed using the canonical ensemble with constant temperature.

\section{Phase diagrams}

From the above potential function, it is possible to derive the phase diagrams depending on the coefficients $A, B, C$ and $H$. The $T-A$ diagrams represented on Fig. 3 have been obtained from the hexagonal model by setting $B$ and $C$ to -2.0 resp. -1.0 . It has been shown elsewhere (Parlinski and Chapuis, 1993) that the magnitude of the wave vector $\mathbf{k}$ associated with a stable incommensurate modulation is directly related with the coefficient $B$. In our example, the value of $B$ corresponds to the wave vector $k=a^{*} / 6$. The phase boundary between the normal $(N)$ and the lock-in phase $(L)$ has been detected from small changes in the average potential energy and the average position $\left\langle z_{j, l, n}\right\rangle$ as a function of the parameter $A$. The two types of incommensurate phases $(I, 1 q)$ and $(I, 3 q)$ can be detected from the intensity of the satellite reflections obtained from the Fourrier transform of the spatial distribution of the particles. The $(I, 1 q)$ type is characterised by one pair of satellites near the main reflections whereas the $(I, 3 q)$ type is characterised by three pairs of satellites close to the main reflections. It is interesting to note that the lock-in and the incommensurate $3 q$ phases appear only for non-zero values of the third order potential term $H$.

The results of the simulation reveal the importance not only of the nearest neighbour interactions but also of the second nearest interactions. Coefficient $B$ controlling the nearest neighbours interactions in the potential function has been set to -2 . The coefficient of the next nearest

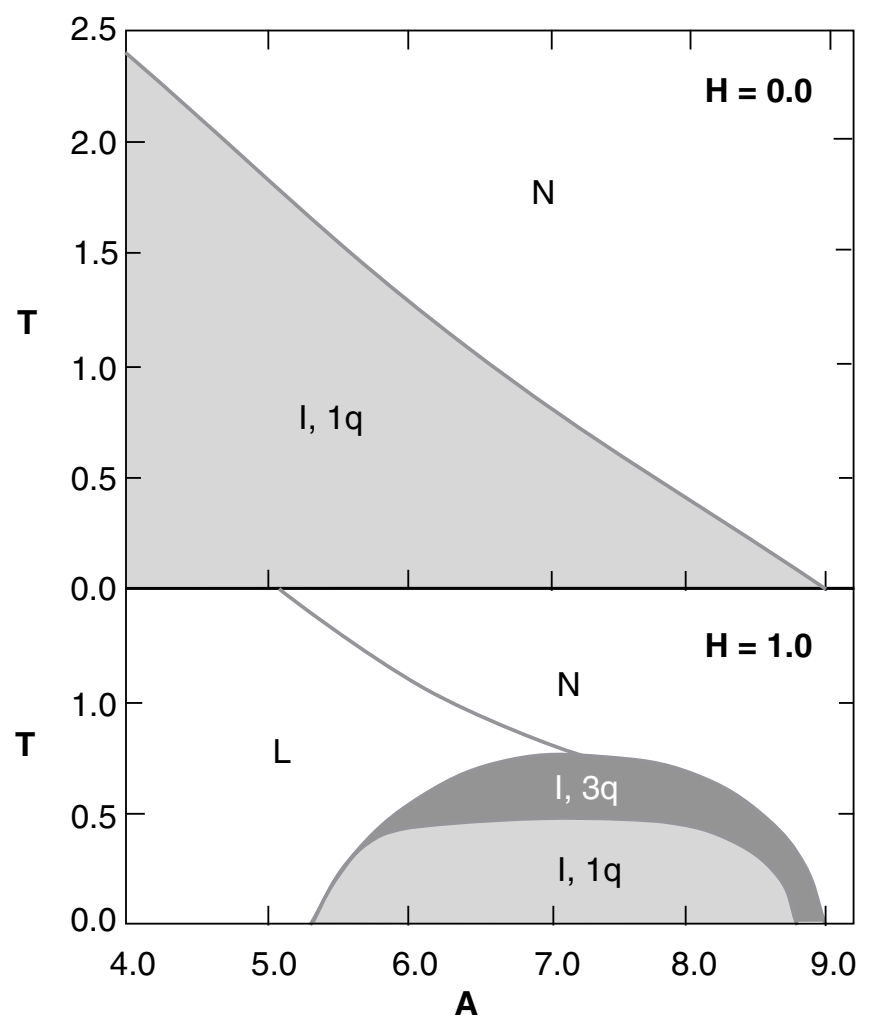

Fig. 3. $T-A$ phase diagrams deduced from the MD simulation with $B=-2$ and $C=-1 . N, I$ and $L$ indicate the normal, incommensurate resp. lockin phases. 
neighbours has been set to 1 (third and fourth line in the potential function terms given in brackets). Thus the existence of the incommensurate phases indicated in the diagram reveal that both type of interactions (nearest and next nearest) must be represented in the potential and moreover with coefficients of roughly the same magnitude. In the next sections, we shall describe two examples of incommensurate structures and show the role of some specific interactions which are responsible for the incommensuration.

\section{$\mathrm{Na}_{2} \mathrm{CO}_{3}$ and its incommensurate phase $\gamma$}

$\gamma-\mathrm{Na}_{2} \mathrm{CO}_{3}$ is a very interesting case study and can be considered as the archetype of an incommensurate crystal. Indeed its structure analysis was at the origin of the concept of superspace development applied to the description of modulated structures. In 1964, Brouns et al. observed the presence of satellite reflections for this compound. Ten years later de Wolff (1974) proposed for the first time the description of a modulated structure in space of higher dimension and in 1976, the same author and coworkers published the incommensurately modulated structure of $\gamma-\mathrm{Na}_{2} \mathrm{CO}_{3}$ in the superspace formalism. The interval between the first observation of satellites and the publication of the incommensurate structure was dedicated to the theoretical development of the superspace concept for one dimensionally modulated structures (de Wolff et al. 1981). We completed the work initiated by de Wolff by taking advantage of modern diffraction equipments in order to improve the resolution of the incommensurate structure and to get some deeper insight into the nature of the incommensurate phase. In our refinement we used satellites of up to $6^{\text {th }}$ order which considerably improved the details of the structure. Moreover, we succeeded to refine the lockin phase stable below $170 \mathrm{~K}$ (Dušek et al., 2003). Table 1 indicates the four phases of $\mathrm{Na}_{2} \mathrm{CO}_{3}$ which have been clearly identified up to this date.

Let us first describe the general features of the $\mathrm{Na}_{2} \mathrm{CO}_{3}$ structure. Fig. 4 is a schematic representation of the structure where all the $\mathrm{O}$ atoms have been omitted. We shall show later that the role of the $\mathrm{O}$ atoms is essentially to insure the charge neutrality bearing little influence on the overall aspect of the architecture. The basic structural element is a graphite or $B N$ type layer consisting of $C$ and $\mathrm{Na} 3$ atoms (Fig. 5). The $\mathrm{O}$ atoms are covalently bound to the $\mathrm{C}$ atoms. In this layer, the $\mathrm{C}-\mathrm{Na}$ distances are close to $3 \AA$ and the hexagons have nearly ideal shape. It is remarkable that the variation of interatomic distances in this layer is negligible between all four phases. The layers are stacked in the third dimension leaving hexagonal channels throughout the structure. Fig. 4 shows that $\mathrm{C}$ and $\mathrm{Na} 3$

Table 1. The sequence of commensurate and incommensurate phases of $\mathrm{Na}_{2} \mathrm{CO}_{3}$ and their transition temperature $(\mathrm{K})$.

\begin{tabular}{|c|c|c|c|c|}
\hline$\alpha$ & $\beta$ & & $\gamma$ & $\delta$ \\
\hline 754 & & 605 & 170 & \\
\hline $\mathrm{PG}_{3} / m m c$ & $C 2 / m$ & & $C 2 / m(\alpha 0 \gamma) 0 s$ & $P 2_{1} / n$ \\
\hline
\end{tabular}

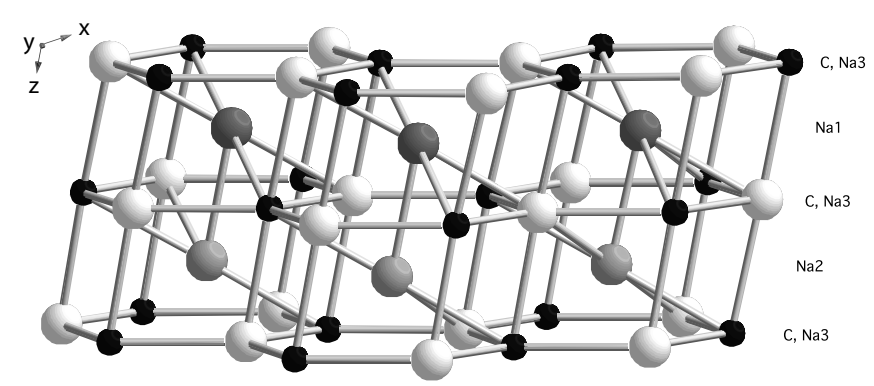

Fig. 4. Structure of $\mathrm{Na}_{2} \mathrm{CO}_{3}$ below $754 \mathrm{~K}$ where all the $\mathrm{O}$ atoms have been omitted. Small black spheres are $\mathrm{C}$ atoms.

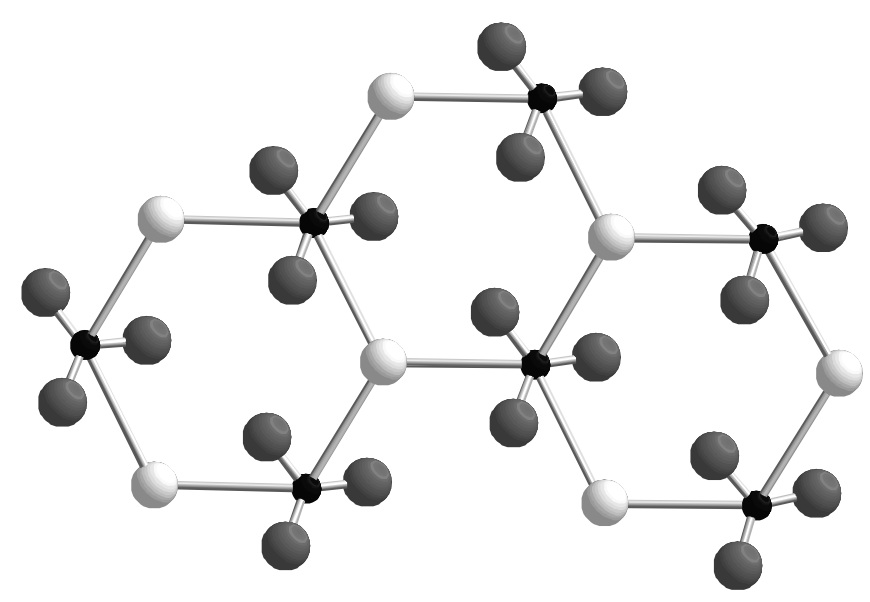

Fig. 5. Essential constituent of the $\mathrm{Na}_{2} \mathrm{CO}_{3}$ structure consisting of a layer of $\mathrm{Na} 3$ and $\mathrm{CO}_{3}$.

atoms alternate in the third dimension. The center of the cavities formed by two parallel hexagons are occupied by additional $\mathrm{Na}$ atoms, $\mathrm{Na} 1$ and $\mathrm{Na} 2$. At high temperature, the $\mathrm{C}-\mathrm{Na} 1,2$ are larger then $3.2 \AA$ and tend to decrease with decreasing temperature to reach finally the optimal value of $3 \AA$. An important architectural entity must be introduced here in order to understand the reasons leading to the phase sequence listed in Table 1 . This entity is the mirror plane $m$ normal to $b$ which contains the three $\mathrm{Na}$ atom types and the $C$ atoms as can be seen from Fig. 4. All atoms of the structure with the exception of a fraction of oxygens are present on this plane.

It is particularly interesting to observe the structure evolution of phase $\beta$ by decreasing temperature until it reaches the incommensurate phase $\gamma$. From the neutron powder diffraction data published by Swainson et al. (1995), we can analyse each structure at a specific temperature and follow the evolution of the close contact formation between $\mathrm{C}$ and $\mathrm{Na}$ atoms. In the high temperature phase $\alpha$, the structure contains three symmetry equivalent mirror planes which intersect along the hexagonal axis. Below the $\alpha$ to $\beta$ transition, only one of the three mirror planes remains $\left(m_{M}\right)$. A description of the content of this plane at different temperatures along with the content of the other two planes $m_{V}$ which are lost during the hexagonal to monoclinic transformation will be sufficient to fully characterise the evolution of the $\mathrm{Na}_{2} \mathrm{CO}_{3}$ phases over the whole temperature range. We can also safely claim that the structure evolution by decreasing temperature is associated with the overall tendency of the system to increase the number of shorter $\mathrm{C}-\mathrm{Na}$ distances on each of these planes. 
The hexagonal to monoclinic transformation can be performed along three different directions. The measurements presented by Dušek et al. (2003) in their Fig. 1 reveal clearly the imprint of the high temperature hexagonal phase $\alpha$ below the transition temperature. One observes that the complete diffraction pattern includes the three twin components as can be derived from symmetry considerations. At the transition temperature, the increase of shorter contacts is achieved by gliding each hexagonal layer relative to each other in one direction. The relative shift of each layer allows the formation of additional shorter $\mathrm{C}-\mathrm{Na} 2$ contacts. The magnitude of this gliding depends on the temperature. From $90^{\circ}$ at the $\alpha$ to $\beta$ transition, this angle reaches finally $99^{\circ}$ at the $\beta$ to $\gamma$ transition. At each step of the structure evolution of the $\beta$ phase deduced from the experimental measurements obtained by decreasing temperature, we observe the step by step formation of short contacts on the $\mathrm{m}_{M}$ plane to reach finally the stable configuration represented on Fig. 6. All the contact distances between atoms indicated on the figures are very close to $3 \AA$. It appears that this unit is preserved at any temperature below the $\beta$ to $\gamma$ phase transition.

The formation of the incommensurate phase $\gamma$ can be much better understood if we concentrate on the structural units formed by the atoms which are located on or close to the $\mathrm{m}_{V}$ planes defined above. These planes can obviously not be realised as perfect mirrors owing to the stable entity of the $m_{M}$ plane content. The monoclinic distortion observed on Fig. 6 has contributed to the stabilisation of the shortest distances in $m_{M}$ plane. Equivalent distortions for the other two $\mathrm{m}_{V}$ planes are consequently not possible. However, the aperiodic character of the phase $\gamma$

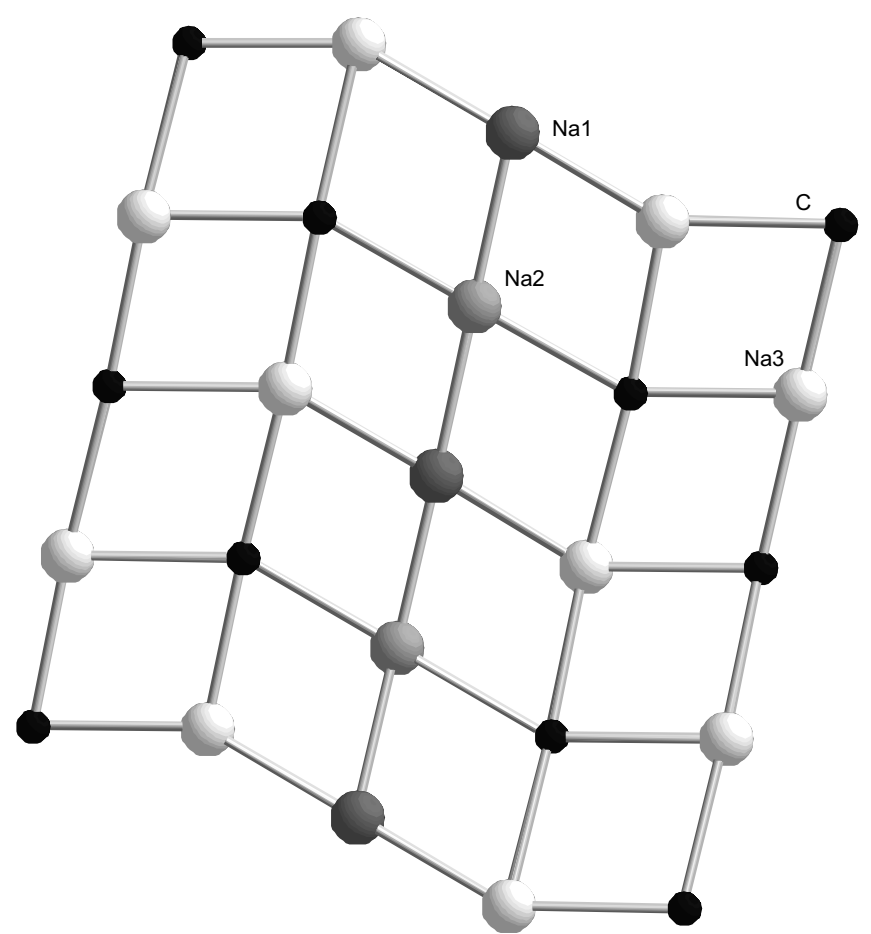

Fig. 6. Structure of atoms located on the $m_{M}$ plane at the $\beta$ to $\gamma$ phase transition. This entity has reached it own stability and remains unchanged by a further decrease of temperature. $\mathrm{O}$ atoms have been omitted. Distances $\leq 3.1 \AA$ are indicated. allows the structure some degrees of freedom to accomodate at least locally in the crystal space structural units which could closely approximate ideal $\mathrm{m}_{V}$ planes. Fig. 7 illustrates the structure of a local fragment of the incommensurate phase $\gamma$ where we observe the tendency of the atoms located on the $m_{V}$ planes to reach an environment similar to the $m_{M}$ plane. This can only be achieved by introducing a considerable amount of distortion in form of a wave along the $z$ direction. This distortion allows a large part of the atoms of the $m_{V}$ planes to approach the ideal contact distances of approximately $3 \AA$.

The comparison of the structural entities extracted from the two phases $\beta$ and $\gamma$ gives some interesting aspects of the incommensurate phase. Fig. 7 illustrates some characteristic structural fragments extracted at various temperatures from the $m_{M}$ plane of phase $\beta$. Each fragment can be matched with a corresponding fragment of the $m_{V}$ plane of phase $\gamma$. Thus, at various values of the internal $t$ parameter, the incommensurate phase reproduces the same structural characteristics observed in phase $\beta$ at various temperatures. This means that the incommensurate structure reproduces locally in different parts of the crystal most of the structural configurations of phase $\beta$ which appear at different temperatures. Thus the formation of the incommensurate structure can be interpreted as an attempt to reproduce at lower temperature, at least locally on the $m_{V}$ planes, the environment found on the $m_{M}$ planes. Owing to the monoclinic deformation, this condition can only be realised in various portions of the crystal which are at first independent (i.e. incommensurate) of the lattice periodicity. The lockin phase $\delta$ stable below $170 \mathrm{~K}$ is commensurate with a structure very similar to phase $\gamma$. The unit cell is a supercell corresponding to the commensurate modulation vector $\mathbf{q}=\frac{1}{6} \mathbf{a}_{1}^{*}+\frac{1}{3} \mathbf{a}_{3}^{*}$. Its structure is characterised by the close $\mathrm{Na}-\mathrm{C}$ and $\mathrm{Na}-\mathrm{Na}$ contacts, which are ordered in 3D space.

In discussing the sequence of phase transitions of $\mathrm{Na}_{2} \mathrm{CO}_{3}$, we have intentionally concentrated on the inter-

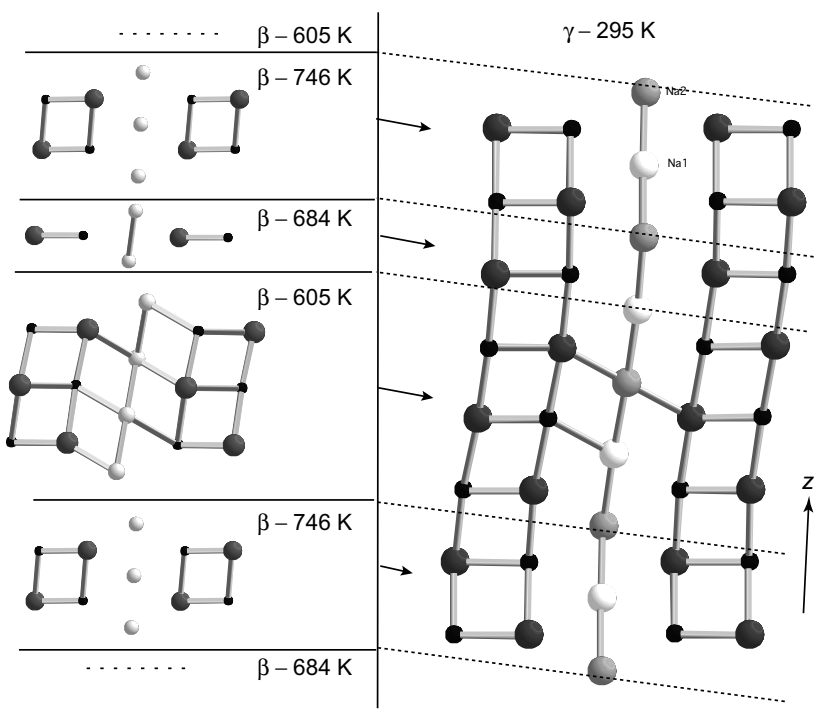

Fig. 7. Comparison of structure fragments extracted from the $m_{M}$ plane of phase $\beta$ at various temperatures with a structure fragment located on the $\mathrm{m}_{V}$ plane of phase $\gamma$ at $295 \mathrm{~K}$. The $\mathrm{O}$ atoms have been omitted. 
actions between non-oxygen atoms. This model is consistent over the complete temperature range from phase $\alpha$ to $\delta$ and does not hint for a fundamental role of the $\mathrm{O}$ atoms other than insuring the electroneutrality. It is however interesting to note that the $\mathrm{CO}_{3}$ unit is not completely rigid. Especially in the phase $\beta$, we observe not only an increase of the $\mathrm{C}-\mathrm{O}$ distances by decreasing temperature, but also a different behaviour depending on the nature of the planes, $m_{V}$ or $m_{M}$ on which the $\mathrm{C}$ atom is located.

\section{The incommensurate structure $\mathrm{K}_{3} \operatorname{In}\left(\mathrm{PO}_{4}\right)_{2}$ and related compounds}

The incommensurate structure of $\mathrm{K}_{3} \mathrm{In}\left(\mathrm{PO}_{4}\right)_{2}$ (Arakcheeva et al., 2003) is closely related to the structure of $\mathrm{Na}_{2} \mathrm{CO}_{3}$ presented in the previous section. The close relationship can best be seen first by omitting the $\mathrm{O}$ atoms. Fig. 8 indicates that the structure can be again described in terms of graphite like layers consisting of $\mathrm{In}, \mathrm{K}$ and $\mathrm{P}$ atoms stacked in the third dimension. The hexagonal channels are occupied by additional $\mathrm{K}$ atoms. Compared to $\mathrm{Na}_{2} \mathrm{CO}_{3}$, this arrangement is less regular and the layers are corrugated. Each $\mathrm{P}$ atom is coordinated by four $\mathrm{O}$ atoms forming a tetrahedron.

By focussing only on the $\mathrm{P}$ atoms, their partial structure consist of a distorted hexagonal close packing. In this packing, the octahedral interstices are occupied by $\mathrm{K}$ atoms whereas the bipyramidal interstices are orderly occupied by $\mathrm{In}$ and $\mathrm{K}$ atoms. The $\mathrm{K}$ atoms located in the bipyramids are shifted towards one of the constituting tetrahedra. The possible displacement of this $\mathrm{K}$ atoms in one of the two tetrahedra lies at the origin of the incommensurate character of the structure represented in Fig. 9.

The geometry of the $\mathrm{P}$ tetrahedra is most stable along the internal coordinate $t$. This indicates that the $\mathrm{P}-\mathrm{O}$ bonds are the strongest ones and do not vary significantly with different local environments. The variability of the $\mathrm{InO}_{6}$-octahedra is higher than for the $\mathrm{InP}_{5}$-bipyramids. The surrounding of $\mathrm{K}$ with $\mathrm{O}$ atoms is extremely variable in distances and $\mathrm{CNs}$, whereas the variety of $\mathrm{K}-\mathrm{P}$ distances in the $\mathrm{KP}_{4}$ tetrahedra is essentially smaller as a function of $t$. The modulation relates to the ordering of the displacement of $\mathrm{K}$ cation within the $\mathrm{KP}_{5}$ bipyramids. The

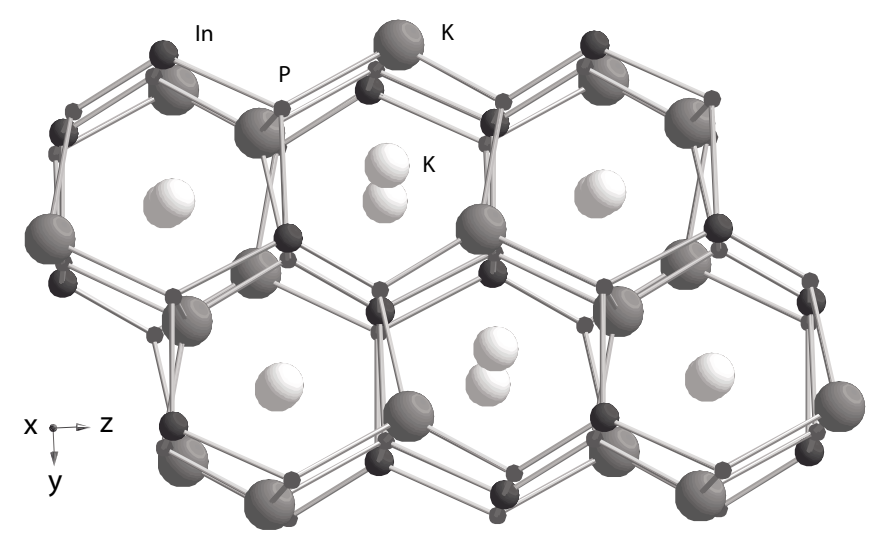

Fig. 8. View of the average structure of $\mathrm{K}_{3} \mathrm{In}\left(\mathrm{PO}_{4}\right)_{2}$ without $\mathrm{O}$ atoms. We can observe the graphite like layers with the channel $\mathrm{K}$ atoms.

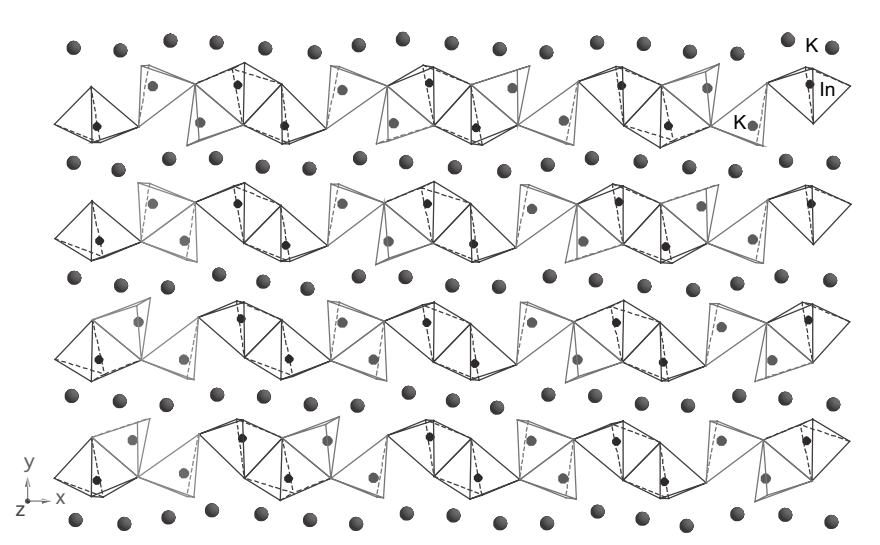

Fig. 9. Portion of the incommensurate structure of $\mathrm{K}_{3} \mathrm{In}\left(\mathrm{PO}_{4}\right)_{2}$ without $\mathrm{O}$ atoms. The dark gray In bipyramids and the light grey $\mathrm{K}$ tetrahedra are coordinated by $\mathrm{P}$ atoms.

driving forces for the modulation of the other cations relates to the $\mathrm{In}-\mathrm{P}$ and $\mathrm{K}-\mathrm{P}$ interactions found in the $\mathrm{InP}_{5}$ bipyramid and $\mathrm{KP}_{6}$-octahedra. The modulation of $\mathrm{O}$ atoms of the rigid $\mathrm{PO}_{4}$ units follows strictly the cations in order to stabilize the first coordination sphere of the In cation, the $\mathrm{InO}_{6}$ octahedron.

The result of the modulated structure refinement allows us to draw some conclusions about the existence and stability of chemical bonds. The analysis of the $\mathrm{InP}_{5}$-bipyramid geometry and the $\mathrm{InO}_{6}$-octahedron geometry indicates that the magnitudes of both interatomic distance and angle variations as functions of $t$ are smaller for the second coordination sphere (the $\mathrm{InP}_{5}$ bipyramid) then for the first (the $\mathrm{InO}_{6}$ octahedron). Consequently, in this incommensurate structure, the In-P interactions are more stable than the $\mathrm{In}-\mathrm{O}$ interactions. The shortest and practically constant In-P distance $(2.84(5) \AA)$ is equal to the covalent $\mathrm{In}-\mathrm{P}$ bond observed in the InP4 tetrahedra of $\mathrm{K}_{3} \mathrm{InP}_{2}$ (Ohse et al., 1993) and $\mathrm{Na}_{3} \mathrm{InP}_{2}$ (Blase et al., 1991). This is a clear indication that the $\mathrm{In}-\mathrm{P}$ interactions observed in these alloys are partially conserved in the oxidized form $\mathrm{K}_{3} \operatorname{In}\left(\mathrm{PO}_{4}\right)_{2}$. One consequence of the short $\mathrm{In}-\mathrm{P}$ distance is that a $\mathrm{PO}_{4}$ tetrahedron and an $\mathrm{InO}_{6}$ octahedron cannot share a single $\mathrm{O}$ atom but only $\mathrm{O}-\mathrm{O}$ edge.

Analogous arguments support the presence of $\mathrm{K}-\mathrm{P}$ interactions in $\mathrm{K}_{3} \operatorname{In}\left(\mathrm{PO}_{4}\right)_{2}$. In $\mathrm{K}_{3} \mathrm{InP}_{2}$, the shortest $\mathrm{K}-\mathrm{P}$ distances lies between 3.25 and $3.28 \AA$. These values compares with the shortest $\mathrm{K}-\mathrm{P}$ distances observed in $\mathrm{K}_{3} \mathrm{In}\left(\mathrm{PO}_{4}\right)_{2}$. Here again, the solution of the incommensurate structure reveal that the second coordination sphere is much more stable in function of $t$. The displacement of the $\mathrm{K}$ atom from the centre of the bipyramid towards one of the tetrahedra can only be explained in terms of the $\mathrm{K}-\mathrm{P}$ interactions. It can thus be safely assumed that both types of interactions $\mathrm{In}-\mathrm{K}$ and $\mathrm{K}-\mathrm{P}$ are the driving forces for the modulation of the structure. This description can be generalised to a larger group of compounds with the general formula $\mathrm{A}_{4-n} \mathrm{R}_{n}\left(\mathrm{XO}_{4}\right)_{2}$ (Arakcheeva et al., 2003). This include in particular $\mathrm{Ni}_{2} \mathrm{In}$ which is usually described as a hexagonal close packing of In atoms with $\mathrm{Ni}$ occupying the octahedral and bipyramidal sites. 


\section{Conclusion}

The superspace formalism which is applied for the description of incommensurate structures has many interesting characteristics which can be exploited in order to analyse not only non-periodic but also periodic structures. From Fig. 1 we have learned that for a specific atom the complete information on all possible displacements from a basic position in the entire crystal space is represented by a periodic curve (string, crenel, sawtooth functions, etc.) which depends on $t$. From the corresponding curves of each atom, it is straightforward to extract any crystal chemical characteristic of interest as a function of $t$. In particular, we can extract the interatomic interactions by observing the relative displacements of $e . g$. groups of atoms as a function of $t$, i.e. in different locations of the crystal space. It is specifically this characteristic which makes the superspace formalism very attractive. As this formalism can also be applied to commensurately modulated structures it is very useful for the study of phase transitions including the comparison of commensurate or incommensurate structures associated with the transition. We have extensively used this possibility for the comparative study of the $\mathrm{Na}_{2} \mathrm{CO}_{3}$ phases presented above.

In order to get some insights into the formation of incommensurate crystal structures, we have applied the methods of molecular dynamics to simulate the conditions for their stability. Based on a hexagonal array of particules where each particle is subject to an unique displacement along the hexagonal axis, we have succeeded to create various commensurate and incommensurate structures and established the conditions for their existence and transitions. These simulations reveal the importance of atomic interactions not only of the nearest neighbours but also of the next nearest neighbours for the formation of incommensurate structures. In addition, the magnitude of the interactions between the next nearest neighbours is of the same magnitude as between the nearest neighbours. We have used this observation to study the origins of the incommensurabilities in two parent compounds $\mathrm{Na}_{2} \mathrm{CO}_{3}$ and $\mathrm{K}_{3} \mathrm{In}\left(\mathrm{PO}_{4}\right)_{2}$. In both compounds, the formation of the incommensurate structures can be linked to interactions between non-O atoms, i.e. between next nearest neighbours. In $\mathrm{Na}_{2} \mathrm{CO}_{3}$, the determining interactions not only for the formation of the incommensurate phase $\gamma$ but also for the formation of the other phases are associated with $\mathrm{Na}-\mathrm{Na}$ and $\mathrm{Na}-\mathrm{C}$ interactions. In $\mathrm{K}_{3} \mathrm{In}\left(\mathrm{PO}_{4}\right)_{2}$, the formation of the incommensurate structure is related to the ordering of $\mathrm{K}$ atoms between two face sharing P-tetrahedra essentially mediated by $\mathrm{K}-\mathrm{P}$ interactions.
This study based on two example of incommensurate structures and on MD simulations can be extended to many other compounds. We can only stress here the role of the superspace formalism to get some deeper insights into the atomic interactions occurring in crystals.

Acknowledgments. This work is supported by the Swiss National Science Foundation, grant No 20-67698.02 which is gratefully acknowledged.

\section{References}

van Aalst, W.; den Holander, J.; Peterse, W. J. A. M.: The modulated structure of $\gamma-\mathrm{Na}_{2} \mathrm{CO}_{3}$ in a harmonic approximation. Acta Cryst. B32 (1976) 47-58.

Arakcheeva, A.; Chapuis, G.; Petř́ícek, V., Dušek, M.; Schönleber, A.: The incommensurate structure of $\mathrm{K}_{3} \mathrm{In}\left(\mathrm{PO}_{4}\right)_{2}$. Acta Cryst. B59 (2003) 17-27.

Blase, W.; Cordier, G.; Somer, M.: Crystal-Structure of Trisodium Tecto-Diphosphidoindate, $\mathrm{Na}_{3} \mathrm{InP}_{2}$. Z. Kristallogr. 195 (1991) 119-120.

Brouns, E.; Visser, J. W.; de Wolff, P. M.: An anomaly in the crystal structure of $\mathrm{Na}_{2} \mathrm{CO}_{3}$. Acta Cryst. 17(5) (1964) 614.

Daniel, V.; Lipson, H.: The dissociation of an alloy of copper, iron and nickel, further X-ray work. Proc. Roy. Soc. London, S.A. 182 (1944) 378-387.

Dušek, M.; Chapuis, G.: Meyer, M.; Petrríček, V.: Sodium carbonate revisited. Acta Cryst. B59 (2003) 337-352.

Hargreaves, M. E.: Modulated structures in some copper\{nickel\{iron alloys. Acta Cryst. 4 (1951) 301-309.

Korekawa, M.: Theorie der Satellitenreflexe. Habilitationsschrift. Ludwigs-Maximilians-Universität zu München. (1967).

Ohse, L.; Somer, M.; Blase, W.; Cordier, G.: Compounds with $\mathrm{SiS}_{2}$ Isoelectronic Anions ${ }^{1}{ }_{8}\left[\mathrm{AlX}^{3-}{ }_{4 / 2}\right]$ and ${ }^{1}{ }_{8}\left[\mathrm{InP}^{3-}{ }_{4 / 2}\right]$ : Synthesis, Crystal-Structures and Vibrational-Spectra of $\mathrm{Na}_{3}\left[\mathrm{AlX}_{2}\right]$, $\mathrm{K}_{2} \mathrm{Na}\left[\mathrm{AlX}_{2}\right]$ and $\mathrm{K}_{3}\left[\mathrm{InP}_{2}\right](\mathrm{X}=\mathrm{P}, \mathrm{As})$. “ $\mathrm{Z}$. Naturforschung $\mathbf{4 8 b}$ (1993) 1027-1034.

Parlinski, K.; Chapuis G.: Mechanisms of phase transitions in a hexagonal model with $1 q$ and $3 q$ incommensurate phases. Phys. Rev. B47 (1993) 13983-13991.

Parlinski, K.; Kwiecinski, S.; Urbanski, A.: Phase-Diagram of a Hexagonal Model with Incommensurate Phases. Phys. Rev. B46 (1992) 5110-5115.

Petříček, V.; Dušek, M.: Methods of structural analysis and computer program Jana2000. Z. Kristallogr. 219 (2004) 692-700.

Swainson, I. P.; Dove, M. T.; Harris, M. J.: Neutron Powder Diffraction Study of the Ferroelastic Phase-Transition and Lattice Melting in Sodium-Carbonate, $\mathrm{Na}_{2} \mathrm{CO}_{3}$. J. Physics-Condensed Matter 7 (1995) 4395-4417.

de Wolff, P. M.: The Pseudo-Symmetry of Modulated Crystal Structures. Acta Cryst. A30 (1974) 777-785.

de Wolff, P. M.: Symmetry operations for displacively modulated structures. Acta Cryst. A33 (1977) 493-497.

de Wolff, P. M.; Janssen, T.; Janner, A.: The Superspace Groups for Incommensurate Crystal-Structures with a One-Dimensional Modulation. Acta Cryst. A37 (1981) 625-636.

Yamamoto, A.: Crystallography of quasiperiodic crystals. Acta Cryst. A52 (1996) 509-560. 